\title{
Fascitis necrotizante como complicación de una osteosíntesis de fractura medial de cadera. Presentación de un caso
}

\author{
Marcos R. Latorre, Fernando Holc, Noelia B. Montenegro, José I. Oñativia \\ Servicio de Ortopedia y Traumatología, Hospital Italiano de Buenos Aires, Ciudad Autónoma de Buenos Aires, Argentina
}

\begin{abstract}
RESUMEN
Introducción: La fascitis necrotizante es un cuadro infrecuente que provoca una alta morbimortalidad. La comunicación de esta entidad asociada a una osteosíntesis de cadera con tornillos canulados es inusual. Se describe el caso de un paciente con múltiples comorbilidades tratado por una fractura medial de cadera mediante una osteosíntesis con tornillos canulados. El paciente ingresó con un cuadro de shock séptico. Requirió cirugía de urgencia con fasciotomía amplia y limpieza quirúrgica más desbridamiento extenso de tejido necrótico. Esta presentación hace hincapié en la importancia de reconocer las principales manifestaciones de esta enfermedad. El diagnóstico es fundamentalmente clínico y requiere de una alta sospecha para instaurar un tratamiento precoz. Conclusión: A pesar de su baja frecuencia y los escasos reportes relacionados con cirugías ortopédicas, es importante tener en cuenta esta entidad como una posible complicación de la cirugía.
\end{abstract}

Palabras clave: Fascitis necrotizante; complicaciones; osteosíntesis.

Nivel de Evidencia: IV

Necrotizing Fasciitis: Complication of Osteosynthesis in Medial Hip Fracture. Case Presentation

\section{ABSTRACT}

Introduction: Necrotizing fasciitis is an infrequent pathology with a high morbidity and mortality. The report of this entity in relation to hip osteosynthesis with cannulated screws is unusual. The aim of this study is to present a case of necrotizing fasciitis as a complication of hip surgery. A case about a patient with multiple comorbidities treated for a femoral neck fracture with osteosynthesis with cannulated screws is described. The patient was admitted to the emergency department of our hospital suffering from septic shock. He required emergency surgical treatment with a wide fasciotomy and a surgical toilet plus extensive debridement of necrotic tissue. The case report emphasizes the importance of recognizing the main manifestations of this disease. The diagnosis is fundamentally clinical and requires high suspicion to establish treatment early. Conclusion: Despite its low frequency and reporting in relation to orthopedic surgeries, it is important to consider this entity as a possible complication of surgery. Necrotizing fasciitis is characterized by its rapid and torpid evolution. Early debridement has been shown to decrease mortality and is the best treatment along with antibiotic therapy.

Key words: Necrotizing fasciitis; complications; osteosynthesis.

Level of Evidence: IV

\section{INTRODUCCIÓN}

La fascitis necrotizante es una rara entidad que provoca una alta morbimortalidad si no se diagnostica a tiempo. Se trata de una infección bacteriana que se caracteriza por afectar principalmente la fascia y el tejido celular subcutáneo, y puede extenderse a los músculos y la piel. El diagnóstico precoz, la administración de antibióticos intravenosos y la rápida intervención quirúrgica son las claves del tratamiento.

Recibido el 3-4-2020. Aceptado luego de la evaluación el 29-6-2020 • Dr. MARCOS R. LATORRE • marcos.latorre @ hospitalitaliano.org.ar (ID) https://orcid.org/0000-0002-2172-2542 Cómo citar este artículo: Latorre MR, Holc F, Montenegro NB, Oñativia JI. Fascitis necrotizante como complicación de una osteosíntesis de fractura medial de cadera. Presentación de un caso. Rev Asoc Argent Ortop Traumatol 2021;86(1):91-98. https://doi.org/10.15417/issn.1852-7434.2021.86.1.1086 
El objetivo de este artículo es presentar un caso de fascitis necrotizante tras una fractura medial de cadera tratada con tornillos canulados. Consideramos importante que, durante el seguimiento posoperatorio, se pueda reconocer y tratar esta complicación precozmente para disminuir la morbimortalidad asociada. Es fundamental conocer sus formas de presentación, los factores de riesgo y su evolución natural para administrar un tratamiento eficaz.

\section{CASO CLÍNICO}

Paciente de 62 años que había sido sometido a una osteosíntesis de cadera derecha por una fractura medial Garden I, en otra institución, 40 días atrás (Figura 1). Ingresa en nuestra central de emergencias derivado por un cuadro de shock séptico. Paciente en plan de trasplante cardiorrenal. Se presenta con nefropatía diabética, hipertensión, miocardiopatía dilatada y coronariopatía. A los 20 días de la cirugía, tiene una celulitis de la herida quirúrgica. Ante la mala respuesta a la antibioticoterapia oral, fue internado para administrarle tratamiento intravenoso.

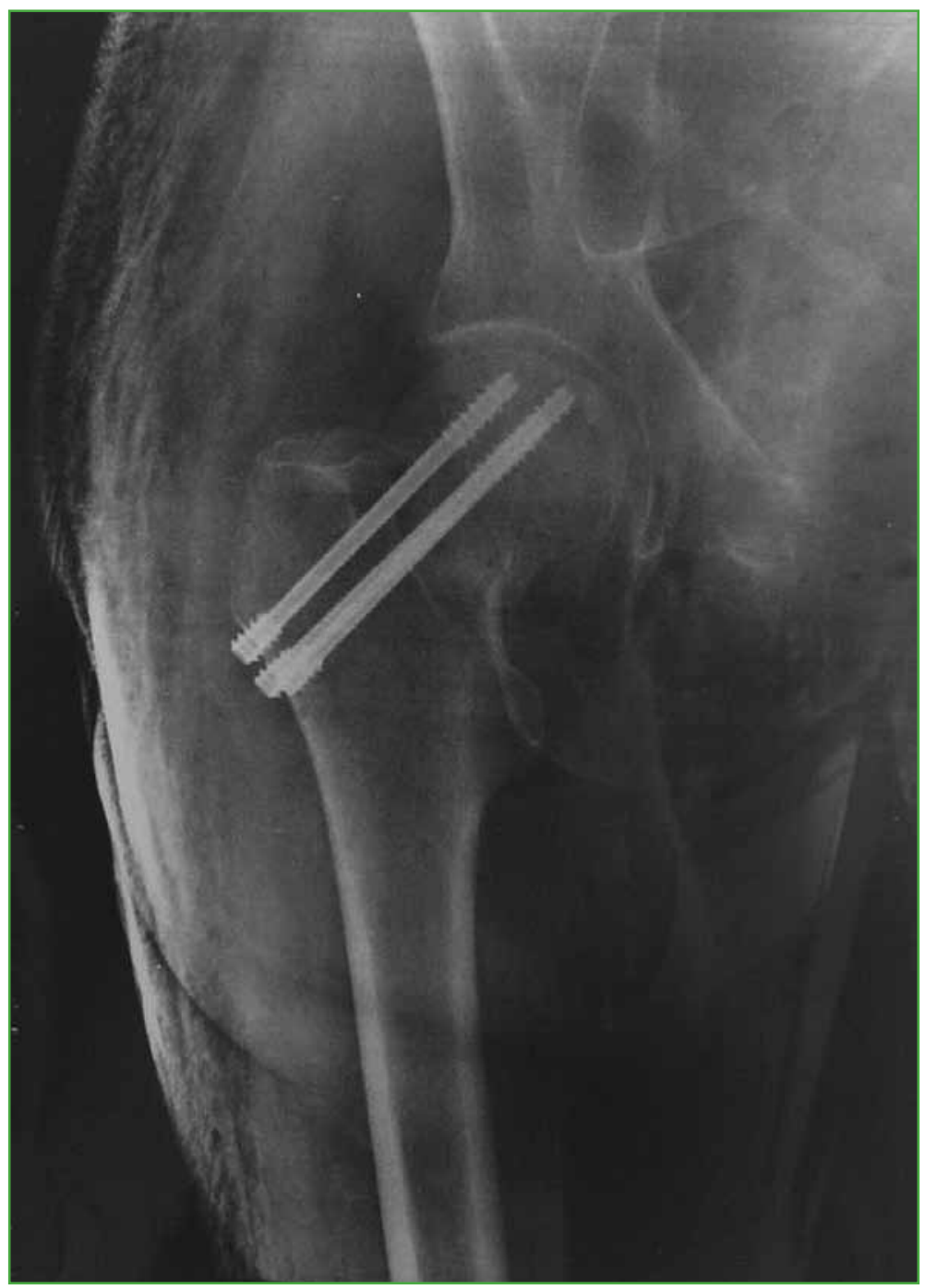

Figura 1. Radiografía anteroposterior de cadera derecha, tomada en el posoperatorio inmediato. 
Al ingresar su estado general es malo, está somnoliento, febril y desorientado en tiempo y espacio. Se constata eritema, calor y crepitantes en la cara lateral del muslo derecho, desde la herida quirúrgica con extensión hacia la región medial de la rodilla (Figura 2).

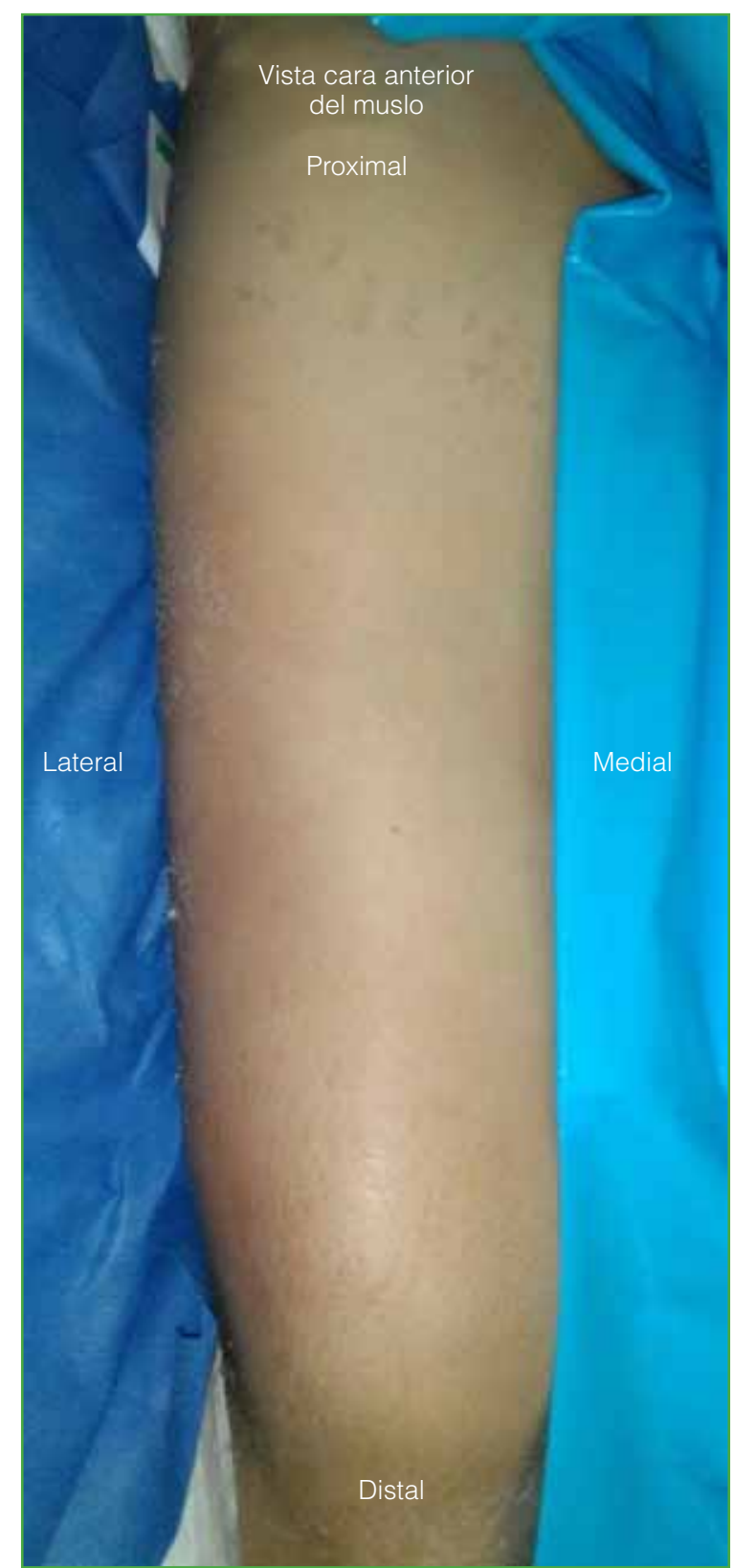

Figura 2. Imagen clínica de la región anterior del muslo cuando el paciente ingresó.

Se toman radiografías y se realiza una tomografía computarizada (Figuras 3 y 4) que muestra imágenes radiolúcidas en las partes blandas, en toda la extensión del muslo. Se indica el tratamiento quirúrgico de urgencia y se administra antibioticoterapia empírica con imipenem y vancomicina. 


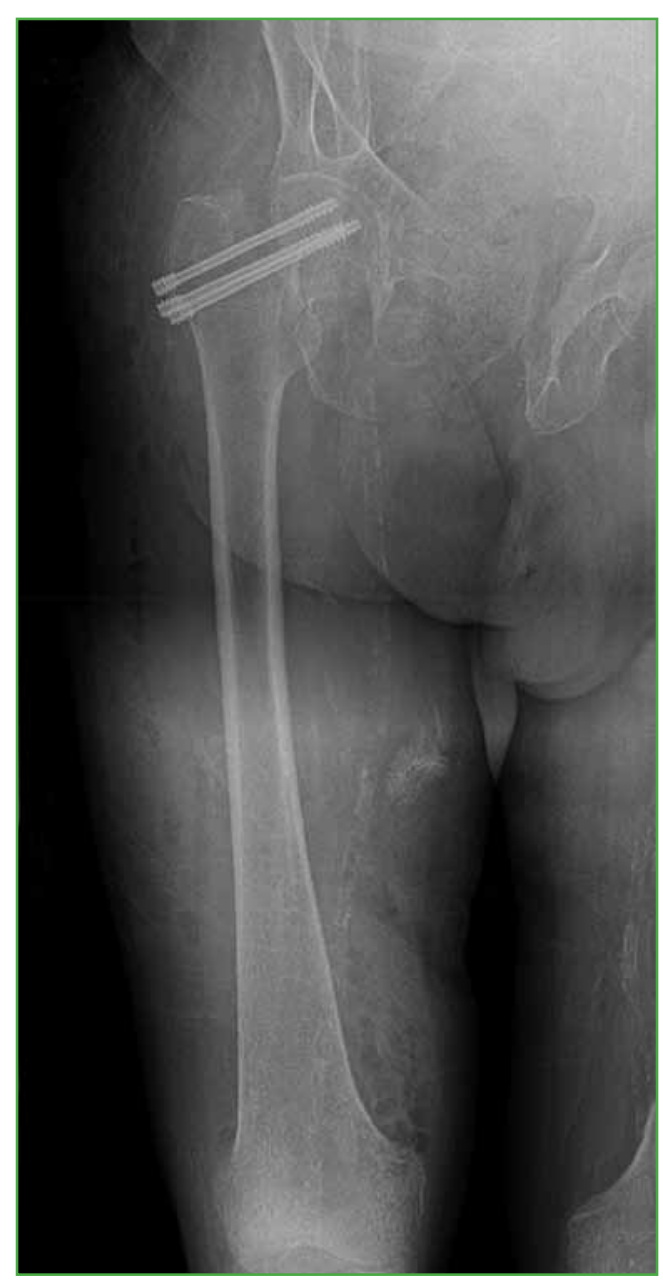

Figura 3. Radiografía anteroposterior de fémur derecho al ingresar. Nótense, además, la pérdida de reducción secundaria de la fractura, las imágenes radiolúcidas que se extienden por las partes blandas de todo el muslo.

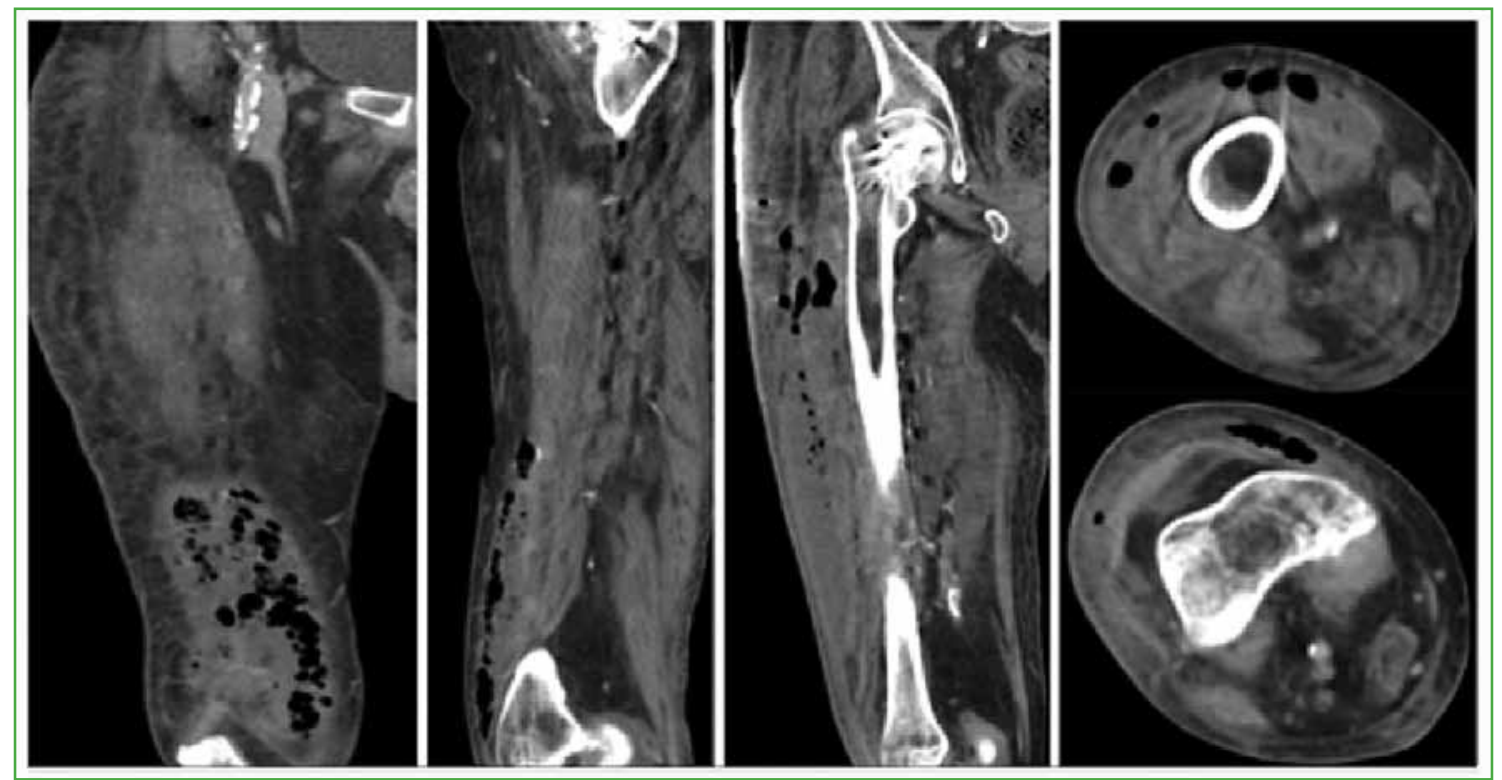

Figura 4. Tomografía computarizada de muslo, cortes coronales y axiales, al ingresar. Se confirma la presencia de aire que se extiende debajo de la fascia. 
La cirugía comienza con un abordaje lateral a nivel de la cicatriz previa, que se extiende al muslo y la cara anteromedial de la rodilla (Figura 5A). En forma espontánea, drena material purulento en toda la extensión y se constata tejido necrótico a nivel muscular y celular subcutáneo (Figura 5B). Se procede a la limpieza con yodopovidona y abundante solución fisiológica, y al desbridamiento del tejido desvitalizado. Se toman muestras de material purulento, interfaz, músculo y hueso de fémur proximal que se envían para cultivo y anatomía patológica. Se realiza una punción articular de la rodilla por un portal anterolateral con instrumental estéril y se obtiene un líquido de características normales, por lo que se decide evitar la artrotomía. Durante el procedimiento, el paciente sufre una descompensación hemodinámica; por lo tanto, se lo da por concluido sin extraer la osteosíntesis.

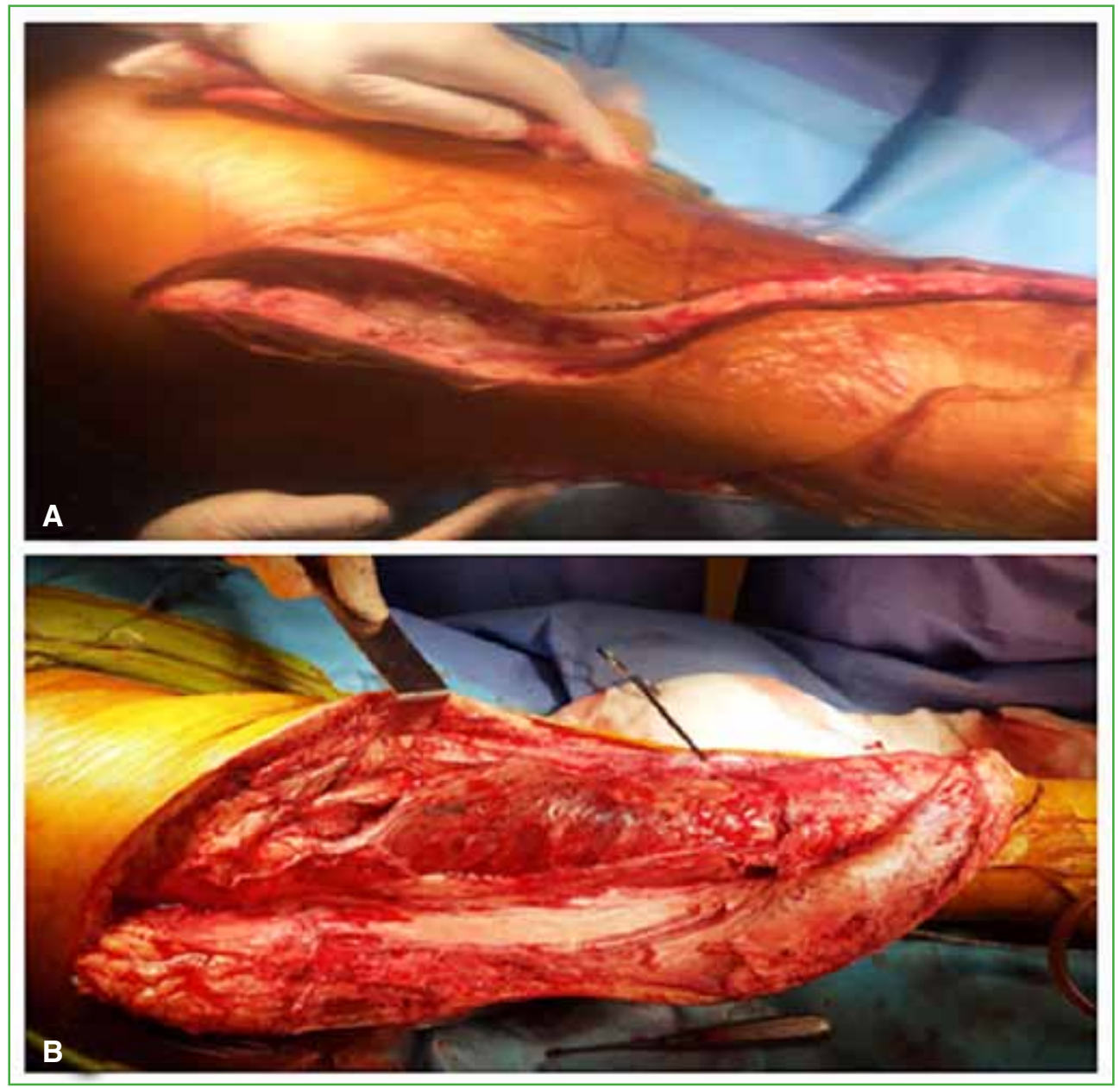

Figura 5. Imágenes clínicas del tratamiento quirúrgico. A. Abordaje lateral sobre la incisión previa extendido a distal, en sentido anteromedial. Se observa material purulento. B. Aspecto luego del desbridamiento extenso hasta obtener tejidos vitales.

En el posoperatorio inmediato, el paciente requirió internación en una unidad de terapia intensiva.

En los cultivos quirúrgicos, se aislaron Klebsiella productora de carbapenemasas y Enterococcus faecalis. El estudio anatomopatológico reveló tejido necrótico y cambios compatibles con osteomielitis aguda. 
A los siete días de la cirugía, se realizó una nueva limpieza más cirugía de Girdlestone y se extrajo el material de osteosíntesis (Figura 6). Se resecó el cartílago articular acetabular y se confeccionó un espaciador disociado de cemento con $2 \mathrm{~g}$ de vancomicina y meropenem, según la sensibilidad de los gérmenes. En la actualidad, el estado general del paciente mejora, está extubado y con descenso progresivo del soporte con inotrópicos.

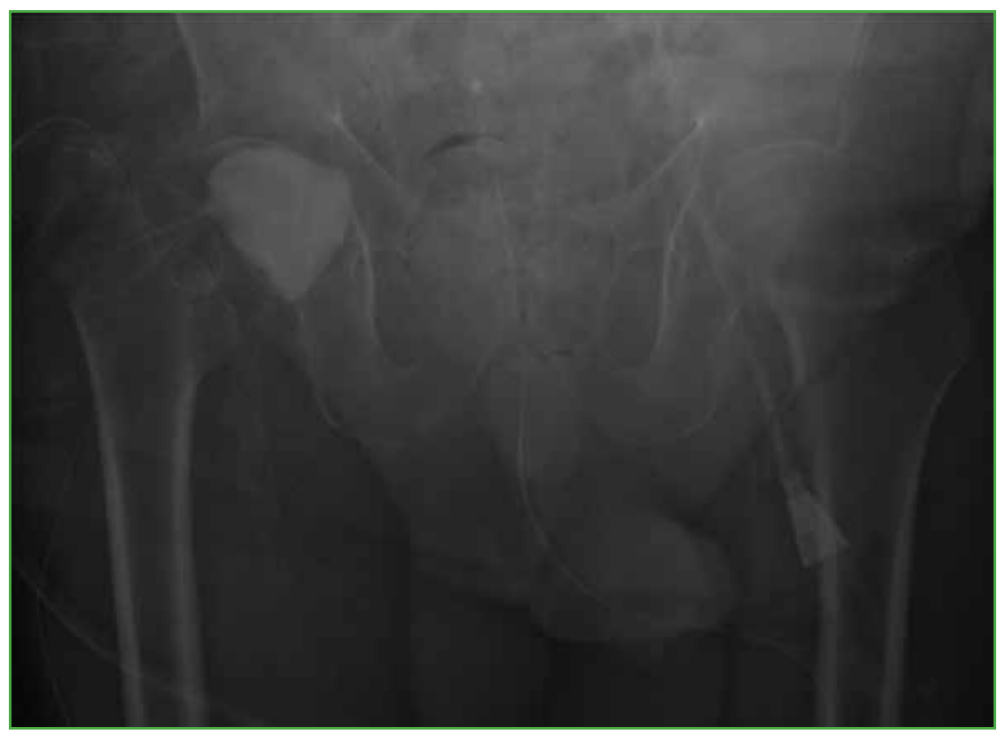

Figura 6. Radiografía anteroposterior de ambas caderas luego del segundo desbridamiento quirúrgico asociado a cirugía de Girdlestone y confección de espaciador de cemento con antibióticos.

\section{DISCUSIÓN}

En los Estados Unidos, la fascitis necrotizante tiene una incidencia de 500 a 1000 casos por año, con una prevalencia de 0,4 casos cada 100.000 habitantes, ${ }^{1}$ y una tasa de mortalidad del 8,7 al 76\%. ${ }^{2}$ Esta enfermedad abarca diversas entidades infecciosas que comprometen las partes blandas y se caracterizan por necrosis de los diferentes tejidos afectados. Como sus principios diagnósticos y terapéuticos son similares, se busca disminuir los tiempos entre la admisión y la cirugía. ${ }^{2}$

Entre los múltiples factores de riesgo de padecer esta entidad, los principales son la diabetes, la inmunosupresión, la enfermedad renal crónica y la cirrosis. ${ }^{3}$

El diagnóstico es principalmente clínico y requiere de una alta sospecha por su amplia variedad de formas de presentación. Se han descrito signos y síntomas locales, como eritema, aumento de la temperatura, induración de la piel y edema, que suelen confundirse con un cuadro de celulitis. El dolor desproporcionado en la lesión cutánea es el síntoma más frecuente (hasta el $100 \%$ de los pacientes). ${ }^{4}$ Con la progresión de la enfermedad subyace el proceso necrótico y aparecen bullas hemorrágicas. El cuadro se acompaña de síntomas generales, como fiebre, hipotensión, taquicardia y alteración de la conciencia.

El puntaje LRINEC (Laboratory Risk Indicator for Necrotizing Fasciitis Score) creado por Wong y cols. ${ }^{5}$ permite diferenciar infecciones necrotizantes de aquellas que no lo son. Se basa en seis variables independientes (proteína $\mathrm{C}$ reactiva, glucosa, leucocitos, sodio, creatinina y hemoglobina) a las que se les otorgan diferentes puntajes. Los puntajes $>6$ tienen un valor predictivo positivo del $92 \%$ y un valor predictivo negativo del $96 \%$ (Tabla). ${ }^{5}$

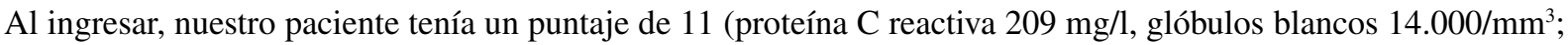
hemoglobina $8,7 \mathrm{~g} / \mathrm{dl}$; sodio $127 \mathrm{mmol} / \mathrm{l}$, creatinina $3,8 \mathrm{mg} / \mathrm{dl}$; glucemia $161 \mathrm{mg} / \mathrm{dl}$ ). 
Tabla. Puntaje LRINEC

\begin{tabular}{|l|l|}
\hline Variable & Puntaje LRINEC \\
\hline Proteína C reactiva, mg/l & \\
\hline$<150$ & 0 \\
\hline$>150$ & 4 \\
\hline Leucocitos, mm $^{3}$ & \\
\hline$<15$ & \\
\hline $15-25$ & 0 \\
\hline$>25$ & 1 \\
\hline Hemoglobina, g/dl & 2 \\
\hline$>13,5$ & \\
\hline $11-13,5$ & 0 \\
\hline$<11$ & 1 \\
\hline Sodio, mmol/l & 2 \\
\hline$\geq 135$ & 2 \\
\hline$<135$ & \\
\hline Creatinina sérica, mg/dl & \\
\hline$\leq 1,6$ & \\
\hline$>1,6$ & \\
\hline Un puntaje $>6$ tiene un valor predictivo positivo del $92 \%$ para el diagnóstico de fascitis necrotizante. \\
\hline$\leq 180$
\end{tabular}

Se han publicado casos de fascitis necrotizante relacionados con osteosíntesis. Cai y cols. ${ }^{6}$ comunicaron un caso por Streptococcus pyogenes que comenzó dos días después de la extracción de una osteosíntesis de radio distal. Shang y cols. ${ }^{7}$ describieron un caso por $S$. aureus sensible a la meticilina, también al segundo día de una osteosíntesis por fractura de tibia. Ambos pacientes fueron tratados mediante múltiples limpiezas, desbridamientos quirúrgicos y antibióticos intravenosos. ${ }^{8}$ No hemos encontrado casos asociados a reducción y osteosíntesis con tornillos canulados en fracturas de cadera, por eso consideramos importante la presentación de este caso.

\section{CONCLUSIONES}

La fascitis necrotizante es un cuadro poco frecuente y son escasas las comunicaciones en relación con cirugías ortopédicas. Sin embargo, es importante tenerla en cuenta en el momento del diagnóstico por su elevada morbimortalidad. La evolución clínica es lo más importante para definir un caso de fascitis necrotizante. Se ha demostrado que el desbridamiento temprano disminuye la mortalidad y es la piedra angular del tratamiento junto con la antibioticoterapia de amplio espectro. 
Conflicto de intereses: Los autores no declaran conflictos de intereses.

ORCID de F. Holc: https://orcid.org/0000-0002-1224-3312

ORCID de N. B. Montenegro: https://orcid.org/0000-0002-5483-9640

ORCID de J. I. Oñativia: https://orcid.org/0000-0001-8534-5101

\section{BIBLIOGRAFÍA}

1. Misiakos EP, Bagias G, Patapis P, Sotiropoulos D, Kanavidis P, Machairas A. Current concepts in the management of necrotizing fasciitis. Front Surg 2014;1:36. https://doi.org/10.3389/fsurg.2014.00036

2. Anaya DA, Dellinger EP. Necrotizing soft-tissue infection: diagnosis and management. Clin Infect Dis 2007;44(5):705-10. https://doi.org/10.1086/511638

3. Yeung YK, Ho ST, Yen CH, Ho PC, Tse WL, Lau YK, et al. Factors affecting mortality in Hong Kong patients with upper limb necrotising fasciitis. Hong Kong Med J 2011;17(2):96-104. PMID: 21471588

4. Young MH, Aronoff DM, Engleberg NC. Necrotizing fasciitis: pathogenesis and treatment. Expert Rev Anti Infect Ther 2005;3(2):279-94. https://doi.org/10.1586/14787210.3.2.279

5. Wong C-H, Khin L-W, Heng K-S, Tan K-C, Low C-O. The LRINEC (Laboratory Risk Indicator for Necrotizing Fasciitis) score: a tool for distinguishing necrotizing fasciitis from other soft tissue infections. Crit Care Med 2004;32(7):1535-41. https://doi.org/10.1097/01.CCM.0000129486.35458.7D

6. Cai Y, Gan Y, Yu C, Tang J, Sun Y. A successful treatment of necrotizing fasciitis following the surgery of distal radius plate removal: A case report and literature review. Medicine (Baltimore) 2018;97(15):e0305. https://doi.org/10.1097/MD.0000000000010305

7. Shang S, Zhang R, Hou Z. Necrotizing fasciitis-A catastrophic complication following routine tibia fracture surgery: A case report and literature review. Medicine (Baltimore) 2017;96(23):e6908. https://doi.org/10.1097/MD.0000000000006908

8. Wong C-H, Chang H-C, Pasupathy S, Khin L-W, Tan J-L, Low C-O. Necrotizing fasciitis: clinical presentation, microbiology, and determinants of mortality. J Bone Joint Surg Am 2003;85-A(8):1454-1460. PMID: 12925624 